\title{
The human element in health care
}

\section{It's no co-incidence that four of the six letters in health are HEAL...Ed northstrum}

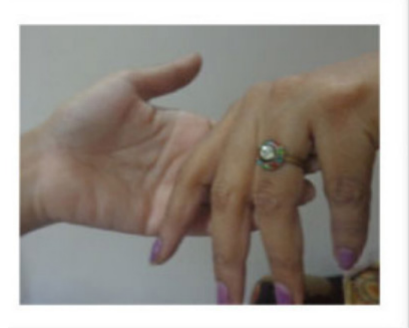

Healing touch

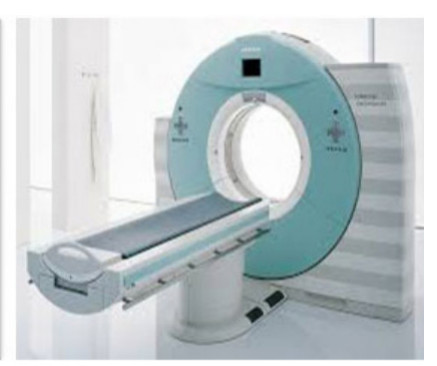

Technology
In this academic presentation we will look into some facets of human element that affects our day-today working of hospital. Understanding of these aspects of human behavior has as much impact on the physical and mental well-being of a healthcare staff members as on the patient's. Healthcare industry is by its inherent nature a service industry that bases its existence on the noble aim of caring for the human life. And therefore, it feels odd when we have to discuss human interface and its value in Healthcare industry. More often than not, one engrosses oneself, in the stress and strain of the working environment so much, as to forget the basic niceties of human interactions \& communication.

To be nice to others in a natural instinct in most human beings. However, in most of them, it may not be expressed spontaneously. Luckily though, it can be easily developed as a acquired skill that can have tremendous mutual benefits to patient and relatives and the caregiver. Generally the etiquettes of behavior and caring attitudes to other humans are collectively called the soft skills.

\section{Stressed person in healthcare setup}

1. General responses to this question are the casualty staff, the intensive care staff, the doctor, the healthcare administrator, and so on.

The patient does figure in the list once in a while. However, it must be realized that patient is the person that:

a. Is suffering from both physical and mental stress.

b. Has come to hospital to get relief from that stress.

c. Has not arrived at hospital on accord of own will. On contrary, coming to hospital (especially as indoor patient) is a stress in itself.

2. On the other hand, a Healthcare worker has:

a. Joined the service on accord of own will and while doing so,

b. Has wilfully, agreed to serve to the best of abilities.

So therefore, at all times, patient's stress and its relief should take precedence over the stress (work related or otherwise) of a Healthcare
Volume 8 Issue 2 - 2017

Joydeep Das Gupta

Work as Honorary Editor - AHPI healthcare E-newsletter, India

Correspondence: Joydeep Das Gupta, Work as Honorary Editor - AHPI healthcare E-newsletter,Association of healthcare Providers India (AHPI), IMA house Indrapastha Marg New Delhi $\square$ II 0002, India, Tel 009I- 98|4994755,

Email authorjoydeepdasgupta@gmail.com

Received: November 26, 2016 | Published: February 03, 2017

worker. There is one more stark reality, which supports the above argument in compelling manner. Patient pays to get relief of stress while healthcare worker gets paid to do so. How blunt! But, how true.

\section{Soft Skills- tools}

3. Soft Skills is not something difficult to practice or totally out of this world! This is what you need to do to practice them.

a. Greet everyone around you with a smile and gentle tone of voice to your colleagues, patients relatives and so on.

b. Share simple pieces of information with patients and relatives which will put them at ease- like guiding them where to sit, tell them how much time it will take before the doctor will see them or investigation/operation will start etc.

c. See that simple necessities of life are available in the waiting areas- likes comfortable seating, drinking water etc.

d. Extend common courtesy - remember you expect these from anyone anytime. It is cruel not to extend them to a patient who is in distress.

e. Provide smiling service- which enhances the healing effect of medication.

In, short, behave in such a way that you would expect to be behaved with, if you were a patient.

\section{Soft skills \& its importance in health care?}

4. It is a fact of life that very few patients know to understand the technical quality of medical treatment that is given to them. They remember a Healthcare setup mainly for two things :

a. Relief of symptoms and the speed with which it was achieved.

b. Behavior of the staff members of the hospital.

5. This contributes mainly to the so-called "Brand Image" and "Good Word of Mouth" in the community. Generally speaking soft skills give over $80 \%$ of Good Word of Mouth while technical superiority contributes $20 \%$ or less. 
6. There is one more huge advantage of being nice to others and keep smiling. It is a known scientific fact that smiling/laughing induces brain to release certain chemicals in our bloodstream that reduces stress, produce feeling of happiness and well-being. Thus, it does a lot of good to your health, too. If you have a nice and pleasant personality!

Now-a-days Hospital Improvement and Management is a critical and extremely important tool needed for efficient process and departmentalization. All important non-medical wing should be present to tackle all such patient facilitation problems as faced by patients \& their accompanied relatives. But in spite of all support department such as Administration, Public Relation, Guest relation \& Front office management, Human Resource management, Housekeeping \& Patient comfort, Sanitation, Bio-medical Engineering, Facility \& Estate, Maintenance \& Engineering etc, we are still lagging behind to provide best holistic care to patient.

Patients need guidance and minute-to-minute holistic gesture during their treatment procedures in the hospital. Supportive family members not able to accompany the patient at each service point To fulfil this important gap we may explore patient co-ordination \& welfare department with staff position of "Patient Co-ordinator" \& "Patient Guide". Said dept will help for better patient service, and for better inter-departmental co-ordination. This department is a very important wing to serve patient with mind. Due to the growing awareness about health amongst the masses, Hospitals all over the world are therefore under tremendous pressure to improve their patient services. Therefore, in the years to come Healthcare Administration \& Management will become more \& more important.
With technological innovations in the medical field, patients have become much more demanding and dependent on technologies that understands the patient, their feelings and emotions. Now-a-days patients' sentiments and expectations are overshadowed by treatments and procedures. The increasing gap between what patients want and what general practitioners perceive as important has resulted in increased dissatisfaction of patients with the health care system. The claim for the measure of satisfaction may be the final common pathway for all health care outcomes. Over a lifetime, patients' expectations of health care may change dramatically. Some patients may place more emphasis on technical competence where as other patients fulfilment of personal needs, comfort, dignity and supportive services will be of paramount importance. Understanding how things look through the patient's eyes should be a central part of any healthcare quality improvement program. Therefore, in order to provide the best patient care with a human touch we should promote human element in healthcare and initiate patient co-ordination concept in each and every health care service unit.

\section{Acknowledgments}

None.

\section{Conflicts of interest}

Author declares there is no conflicts opf interest.

\section{Funding}

None. 